\title{
A Note on the Galois Brumer-Stark Conjecture
}

\author{
Jiro NOMURA
}

Keio University

(Communicated by K. Matsuno)

\begin{abstract}
In this paper, we prove, for primes $l$ satisfying some conditions, the $l$-parts of the Galois BrumerStark conjecture, which is formulated by Dejou and Roblot for Galois CM-extensions with dihedral or generalized quaternion Galois group of specified degrees.
\end{abstract}

\section{Introduction}

In number theory, the study of the mysterious relation between the special values of $L$-functions and arithmetic objects has been a main subject. Concerning this subject, the Brumer-Stark conjecture has been studied for many years. This conjecture is formulated for abelian extensions of number fields and concerned with the Galois module structure of ideal class groups. There exists a large body of evidence in support of this conjecture, see, for example, [6], [9] and [5].

In the last several years, non-abelian generalizations of the Brumer-Stark conjecture have been formulated independently by D. Burns [1], [2] and A. Nickel [10]. On the other hand, in [4], G. Dejou and X.-F. Roblot formulated another non-abelian generalization, the "Galois Brumer-Stark conjecture", which does not seem to be equivalent, in general, to the conjectures by Burns and Nickel. An important difference between the Galois Brumer-Stark conjecture and conjectures by Burns and Nickel is that the former has a claim concerning a field extension but the latter do not have such a claim. The main result of this paper is a proof of the Galois Brumer-Stark conjecture in a special case.

We briefly review the formulation here. Let $K / k$ be a finite Galois extension of number fields with Galois group $G$ and $S$ a finite set of places of $k$ which contains all infinite places and all finite places which ramify in $K$. Then the "Stickelberger element" $\theta_{K / k, S}$ is defined by using the special values of $L$-functions attached to $K / k$, for the precise definition, see $\S 3$.

Received February 12, 2015; revised April 27, 2015

Mathematics Subject Classification: 11R29, 11R42

Key words and phrases: Brumer-Stark conjecture, equivariant $L$-values, class groups

The author is partially supported by JSPS Core-to-core program, "Foundation of a Global Research Cooperative Center in Mathematics focused on Number Theory and Geometry". 
We set

$$
K^{\circ}:=\left\{\alpha \in K^{*}|| \alpha^{\sigma} \mid=1 \text {, for all embeddings } \sigma: K \hookrightarrow \mathbf{C}\right\} .
$$

Moreover, we write $\mu(K), w_{K}$ and $\operatorname{Ann}_{\mathbf{Z}[G]}(\mu(K))$ for the group of roots of unity in $K$, the order of $\mu(K)$ and $\mathbf{Z}[G]$-left annihilator of $\mu(K)$, respectively. Finally, we define the natural number $d_{G}$ to be the least common multiple among the order of the commutator subgroup of $G$ and the cardinalities of the conjugacy classes of $G$. Then the following is the statement of the Galois Brumer-Stark conjecture:

Conjecture 1.1. Assume $|S| \geq 2$. Then

- $d_{G} \operatorname{Ann}_{\mathbf{Z}[G]}(\mu(K)) \theta_{K / k, S} \subset \mathbf{Z}[G]$.

- For each fractional ideal $\mathfrak{A}$ of $K$, there exists $\alpha \in K^{\circ}$ such that

$$
\mathfrak{A}^{d_{G} w_{K} \theta_{K / k, S}}=(\alpha),
$$

and $K\left(\alpha^{1 / w_{K}}\right) / k$ is a "strong central extension" of $K / k$.

For the definition of the strong central extension, see $\$ 4$. For a prime number $p$, we can formulate the following " $p$-part" of the above conjecture:

CONJECTURE 1.2. Assume $|S| \geq 2$. Then

- $d_{G} \operatorname{Ann}_{\mathbf{Z}[G]}(\mu(K)) \theta_{K / k, S} \subset \mathbf{Z}[G]$.

- For each fractional ideal $\mathfrak{A}$ of $K$ whose class in the ideal class group of $K$ has p-power order, there exists $\alpha \in K^{\circ}$ such that

$$
\mathfrak{A}^{d_{G} w_{K} \theta_{K / k, S}}=(\alpha),
$$

and $K\left(\alpha^{1 / w_{K, p}}\right) / k$ is a strong central extension of $K / k$, where $w_{K, p}$ is the order of $\mu(K) \otimes \mathbf{Z}_{p}$.

We easily see that Conjecture 1.1 is true if and only if Conjecture 1.2 is true for all primes $p$.

In what follows we assume $K / k$ is a finite Galois CM-extension, that is, $k$ is totally real and $K$ is an imaginary quadratic extension of some totally real field. For an odd prime $p$ and a nonzero natural number $n$, we write $D_{4 p}$ and $Q_{2^{n+2}}$ for the dihedral group of order $4 p$ and the generalized quaternion group of order $2^{n+2}$, respectively. Then the main results of this paper are the followings, which are Theorems 5.4 and 5.8 in $\$ 5$.

TheOrem 1.3. Assume $G$ is isomorphic to $D_{4 p}$. Then the l-part of the Galois Brumer-Stark conjecture is true for $K / k, S$, where $l$ is an odd prime whose mod p class generates $(\mathbf{Z} / p \mathbf{Z})^{*}$. Further if $K$ does not contain p-power roots of unity, the p-part of the Galois Brumer-Stark conjecture is also true for $K / k$ and $S$.

THEOREM 1.4. Assume $G$ is isomorphic to $Q_{2^{n+2}}$. Then the 2-part of the Galois Brumer-Stark conjecture is true for $K / k$ and $S$. 
The keys of the proofs of these theorems are [11, Propositions 5.3 and 5.8] (in fact we need slight refinements of [11, Propositions 5.3 and 5.8], which are Propositions 5.2 and 5.6 in $\S \S 5.1$ and 5.2, respectively). These propositions were also needed to prove the "weak non-abelian Brumer-Stark conjecture" by Nickel under the same assumption as above [11, Theorems 5.1 and 5.6]. Therefore the author thinks it is a very interesting problem to find a relation between two conjectures, however, we do not study the problem in this paper.

Notation. For a finite group $G$, we write $\operatorname{Irr} G$ and $[G, G]$ for the set of $\mathbf{C}$-valued irreducible characters of $G$ and the commutator subgroup of $G$, respectively. If $G$ is the Galois group of a CM-extension of number fields, we write $\operatorname{Irr}^{-} G$ for the subset of $\operatorname{Irr} G$ consisting of the characters $\chi$ such that $\chi(j)=-\chi(1)$ for the unique complex conjugation $j$ of $G$.

For a number field $F$ and a prime number $p$, we write $\mu(F), w_{F}, w_{F, p}$ and $C l(K)$ for the group of roots of unity in $F$, the order of $\mu(F)$, that of $\mu(F) \otimes \mathbf{Z}_{p}$ and the ideal class group of $F$, respectively. For a nonzero natural number $n$, we fix a primitive $n$-th root of unity $\zeta_{n}$ in a fixed algebraic closure $\overline{\mathbf{Q}}$ of $\mathbf{Q}$.

For a finite Galois extension $K / k$ of number fields with Galois group $G$, we write $S_{\infty}$, $S_{\text {ram }}$ and $K^{\mathrm{ab}}$ for the set of all infinite places of $k$, the set of all finite places of $k$ which ramify in $K$ and the maximal abelian subextension of $K / k$ i.e. $K^{a b}=K^{[G, G]}$, respectively.

\section{Central conductors}

Let $G$ be a finite group. We set

$$
e_{\chi}:=\frac{\chi(1)}{|G|} \sum_{\sigma \in G} \chi(\sigma) \sigma^{-1}, \quad \operatorname{pr}_{\chi}:=\frac{|G|}{\chi(1)} e_{\chi}=\sum_{\sigma \in G} \chi(\sigma) \sigma^{-1}, \quad \chi \in \operatorname{Irr} G .
$$

Then $e_{\chi}$ is a central primitive idempotent of $\mathbf{C}[G]$ and $\mathrm{pr}_{\chi}$ is the associated projector.

We choose a maximal $\mathbf{Z}$-order $\mathfrak{m}(G)$ in $\mathbf{Q}[G]$ which contains $\mathbf{Z}[G]$. Then the central conductor $\mathfrak{F}(G)$ of $\mathfrak{m}(G)$ over $\mathbf{Z}[G]$ is given by

$$
\mathfrak{F}(G):=\{x \in \zeta(\mathbf{Z}[G]) \mid x \mathfrak{m}(G) \subset \mathbf{Z}[G]\},
$$

where $\zeta(\mathbf{Z}[G])$ is the center of $\mathbf{Z}[G]$. By Jacobinski's central conductor formula ([7, Theorem 3] also see [3, §27]), we have

$$
\mathfrak{F}(G) \cong \bigoplus_{\chi \in \operatorname{Irr} G / \sim} \frac{|G|}{\chi(1)} \mathfrak{D}^{-1}(\mathbf{Q}(\chi) / \mathbf{Q})
$$

where $\mathbf{Q}(\chi)=\mathbf{Q}(\chi(g) ; g \in G), \mathfrak{D}^{-1}(\mathbf{Q}(\chi) / \mathbf{Q})$ is the inverse different of $\mathbf{Q}(\chi)$ over $\mathbf{Q}$ and the direct sum runs over the irreducible characters of $G$ modulo $\operatorname{Gal}(\overline{\mathbf{Q}} / \mathbf{Q})$-action. From this formula, we see that elements $x$ in $\mathfrak{F}(G)$ are of the form

$$
x=\sum_{\chi \in \operatorname{Irr} G / \sim \sigma \in \operatorname{Gal}(\mathbf{Q}(\chi) / \mathbf{Q})} x_{\chi}^{\sigma} \operatorname{pr}_{\chi^{\sigma}}, x_{\chi} \in \mathfrak{D}^{-1}(\mathbf{Q}(\chi) / \mathbf{Q}) .
$$




\section{Stickelberger elements}

Let $K / k$ be a finite Galois extension of number fields with Galois group $G$ and $S$ a finite set of places of $k$ which contains $S_{\text {ram }}$ and $S_{\infty}$. We define the Stickelberger element for $K / k$ and $S$ by

$$
\theta_{K / k, S}:=\sum_{\chi \in \operatorname{Irr} G} L_{S}(K / k, \check{\chi}, 0) e_{\chi} \in \zeta(\mathbf{C}[G]),
$$

where $L_{S}(K / k, \check{\chi}, s)$ is the $S$-truncated Artin $L$-function attached to $\check{\chi}$ and $\check{\chi}$ is the contragredient character of $\chi$. This element actually belongs to $\zeta(\mathbf{Q}[G])$ by the rank zero Stark conjecture, which is a theorem of Siegel and Klingen for abelian extensions, and is proved by Brauer induction for general extensions [13, Theorem 1.2]. If $S=S_{\text {ram }} \cup S_{\infty}$, we abbreviate $\theta_{K / k, S}$ by $\theta_{K / k}$.

Let $m_{G}$ be the least common multiple of the cardinalities of the conjugacy classes of $G$, and let $s_{G}$ be the order of the commutator subgroup of $G$. We set $d_{G}:=1 . \operatorname{c.m}\left\{m_{G}, s_{G}\right\}$. Then in [4] Dejou and Roblot formulated the following conjecture concerning the integrality of Stickelberger elements.

CONJECTURE $3.1(\operatorname{Int}(K / k, S))$.

$$
d_{G} \operatorname{Ann}_{\mathbf{Z}[G]}(\mu(K)) \theta_{K / k, S} \subset \mathbf{Z}[G] .
$$

REMARK 3.2. In [10] Nickel formulated another integrality conjecture, which predicts that " $T$-modified" Stickelberger elements $\theta_{K / k, S}^{T}=\delta_{T} \theta_{K / k, S}$ belong to $\mathcal{I}(G)$. Here $\mathcal{I}(G)$ is the module over $\zeta(\mathbf{Z}[G])$ generated by the reduced norms of matrices over $\mathbf{Z}[G]$ and $\delta_{T}$ is an element in $\mathcal{I}(G)$. Let $\mathcal{H}(G)$ be the "denominator ideal" defined in [8] such that $\mathcal{H}(G) \mathcal{I}(G) \subset \zeta(\mathbf{Z}[\mathbf{G}])$. Then we have $\mathcal{H}(G) \delta_{T} \subset \operatorname{Ann}_{\zeta(\mathbf{Z}[G])}(\mu(K))$ (cf. [10, Remark 1. ii)] and the proof of [4, Proposition A.1]). Therefore, we see that if Conjecture 3.1 is true, $d_{G} \mathcal{H}(G) \delta_{T} \theta_{K / k, S} \subset \zeta(\mathbf{Z}[G])$. However, it is not clear whether Conjecture 3.1 implies Nickel's integrality conjecture itself. Conversely, if Nickel's integrality conjecture is true, we have $\mathcal{H}(G) \delta_{T} \theta_{K / k, S} \subset \zeta(\mathbf{Z}[G])$ and hence $d_{G} \mathcal{H}(G) \delta_{T} \theta_{K / k, S} \subset \zeta(\mathbf{Z}[G])$. This gives stronger results than Conjecture 3.1 for the elements of the form $\mathcal{H}(G) \delta_{T}$. However, it seems that Nickel's integrality conjecture can not imply the full statement of Conjecture 3.1 since it is concerned only with central elements.

In [4] the above conjecture was proved in some cases, for example, the following was proved.

THEOREM 3.3 ([4], Theorem 7.1 and Proposition 7.3). We set

$$
\theta_{K / k, S}^{>1}:=\left(1-\frac{1}{s_{G}} N_{[G, G]}\right) \theta_{K / k, S}
$$

and assume $G$ has an abelian normal subgroup $H$ of prime index. We write $S_{H}$ for the set of 
places of $K^{H}$ above $S$. Then we have

$$
\theta_{K / k, S}^{>1}=\left(1-\frac{1}{s_{G}} N_{[G, G]}\right) \theta_{K / K^{H}, S_{H}}
$$

and $\operatorname{Int}(K / k, S)$ is true for $K / k$ and $S$.

\section{The Galois Brumer-Stark conjecture}

In this section we review the formulation of the Galois Brumer-Stark conjecture. For the details, see the original paper [4].

Let $K / k$ be a finite Galois extension with Galois group $G$. Then in [4] they introduce the notion "strong central extension", which is defined as follows.

Definition 4.1 ([4]). A finite field extension $L / k$ is a strong central extension of $K / k$ if $L$ contains $K, L / k$ is a Galois extension with Galois group $\Gamma$ and $[\Gamma, \Gamma] \cap$ $\operatorname{Gal}(L / K)=1$.

Now we state the Galois Brumer-Stark conjecture.

CONJECTURE $4.2\left(B S_{\mathrm{Gal}}(K / k, S)\right)$. Let $K / k$ be a finite Galois extension and $S$ a finite set of places which contains $S_{\text {ram }}$ and $S_{\infty}$ with $|S| \geq 2$. Then $\operatorname{Int}(K / k, S)$ is true, and for each fractional ideal $\mathfrak{A}$ of $K$, there exists $\alpha$ in $K^{\circ}$ such that $\mathfrak{A}^{d_{G} w_{K} \theta_{K / k, S}}=(\alpha)$ and $K\left(\alpha^{1 / w_{K}}\right) / k$ is a strong central extension of $K / k$.

For each prime $p$, we get the $p$-part of the conjecture $B S_{\mathrm{Gal}}(K / k, S, p)$ by replacing $w_{k}$ and $\mathfrak{A}$ by $w_{K, p}$ and $\mathfrak{A}$ whose class in $C l(K)$ has $p$-power order, respectively. We see that $B S_{\mathrm{Gal}}(K / k, S)$ is true if and only if $B S_{\mathrm{Gal}}(K / k, S, p)$ is true for all primes $p$.

A peculiarity of the Galois Brumer-Stark conjecture is that the conjecture decomposes into the "abelian part" and the "non-abelian part". More precisely, in [4] they proved the following proposition.

THEOREM 4.3 ([4], Theorem 6.3). Assume that $\operatorname{Int}(K / k, S)$ and $B S\left(K^{\mathrm{ab}} / k, S\right)$ are true. Then $B S_{\mathrm{Gal}}(K / k, S)$ is true if and only iffor any fractional ideal $\mathfrak{A}$ of $K, \mathfrak{A}^{d_{G} w_{K} \theta_{K / k, S}^{>1}}=$ ( $\beta$ ) for some $\beta$ in $K^{\circ}$ and $K\left(\beta^{1 / w_{K}}\right) / k$ is a strong central extension of $K / k$.

By the same proof of the above result in [4], we get the following.

THEOREM 4.4. The same statement as Theorem 4.3 holds with $B S\left(K^{\mathrm{ab}} / k, S\right)$, $B S_{\mathrm{Gal}}(K / k, S)$ and $\beta^{1 / w_{K}}$ replaced by $B S\left(K^{\mathrm{ab}} / k, S, p\right), B S_{\mathrm{Gal}}(K / k, S, p)$ and $\beta^{1 / w_{K, p}}$, respectively.

To prove our main results, we essentially use the above theorem.

\section{Main results}

In this section, we prove our main results Theorems 1.3 and 1.4. 
5.1. Extensions with group $D_{4 p}$. Let $p$ be an odd prime. In this subsection, we prove our main result for a CM-extension whose Galois group $G$ is isomorphic to the dihedral group $D_{4 p}$ of order $4 p$. We use the presentation $D_{4 p}=\left\langle x, y \mid x^{2 p}=y^{2}=1, y x y^{-1}=x^{-1}\right\rangle$. Then the center of $D_{4 p}$ is $\left\{1, x^{p}\right\}$.

5.1.1. Calculation of $d_{G}$ and Stickelberger elements. Conjugacy classes of $D_{4 p}$ are $\{1\},\left\{x^{p}\right\},\left\{x^{2 k} y\right\}_{1 \leq k \leq p},\left\{x^{2 k-1} y\right\}_{1 \leq k \leq p}$ and $\left\{x^{l}, x^{-l}\right\}$, where $1 \leq l \leq p-1$. Then we have

$$
\#\{1\}=\#\left\{x^{p}\right\}=1, \quad \#\left\{x^{l}, x^{-l}\right\}=2 \quad \text { and } \quad \#\left\{x^{2 k} y\right\}_{1 \leq k \leq p}=\#\left\{x^{2 k-1} y\right\}_{1 \leq k \leq p}=p .
$$

Moreover, the commutator subgroup of $D_{4 p}$ is the cyclic group $\left\langle x^{2}\right\rangle$ of order $p$. Combining these facts, we have

$$
d_{G}=1 . \operatorname{com}\{1,2, p\}=2 p .
$$

LemMA 5.1. Let $K / k$ be a finite Galois CM-extension of number fields whose Galois group is isomorphic to $D_{4 p}$ and let $S$ be a finite set of places of $k$ which contains $S_{\text {ram }}$ and $S_{\infty}$. We set $H:=\langle x\rangle$. Then

$$
d_{G} w_{K} \theta_{K / k, S}^{>1}=\left(\sum_{\chi \in \operatorname{Irr}^{-}} \operatorname{pr}_{\chi}\right) w_{K} \theta_{K / K^{H}, S_{H}} .
$$

Proof. Regarding $d_{G}$ as an element in $\mathbf{C}[G]$, we see that

$$
\begin{aligned}
d_{G}=2 p & =\sum_{\chi \in \operatorname{Irr} G} 2 p e_{\chi} \\
& =\sum_{\chi \in \operatorname{Irr} G, \chi(1)=1} 2 p\left(\frac{1}{4 p} \mathrm{pr}_{\chi}\right)+\sum_{\chi \in \operatorname{Irr} G, \chi(1)=2} 2 p\left(\frac{1}{2 p} \mathrm{pr}_{\chi}\right) \\
& =\sum_{\chi \in \operatorname{Irr}} \frac{1}{G, \chi(1)=1} \operatorname{pr}_{\chi}+\sum_{\chi \in \operatorname{Irr} G, \chi(1)=2} \operatorname{pr}_{\chi} .
\end{aligned}
$$

Then we have by Theorem 3.3

$$
\begin{aligned}
d_{G} w_{K} \theta_{K / k, S}^{>1} & =\left(\sum_{\chi \in \operatorname{Irr} G, \chi(1)=1} \frac{1}{2} \mathrm{pr}_{\chi}+\sum_{\chi \in \operatorname{Irr} G, \chi(1)=2} \mathrm{pr}_{\chi}\right) w_{K}\left(1-\frac{1}{s_{G}} N_{[G, G]}\right) \theta_{K / K^{H}, S_{H}} \\
& =\left(\sum_{\chi \in \operatorname{Irr} G, \chi(1)=2} \operatorname{pr}_{\chi}\right) w_{K} \theta_{K / K^{H}, S_{H}} \\
& =\left(\sum_{\chi \in \operatorname{Irr}^{-} G, \chi(1)=2} \operatorname{pr}_{\chi}\right) w_{K} \theta_{K / K^{H}, S_{H}} .
\end{aligned}
$$

The last equality follows since we have

$$
\operatorname{pr}_{\chi} w_{K} \theta_{K / K^{H}, S_{H}}=\operatorname{pr}_{\chi} d_{G} w_{K} \theta_{K / k, S}^{>1}=d_{G} w_{K} L_{S}(K / k, \check{\chi}, 0) \operatorname{pr}_{\chi}
$$


for $\chi \in \operatorname{Irr} G$ with $\chi(1)=2$ and $L_{S}(K / k, \check{\chi}, 0)=0$ if $\chi$ does not belongs to $\operatorname{Irr}^{-} G$.

5.1.2. Main result. In this section we prove Theorem 5.4. In [11] the author proved the following result.

Proposition 5.2 ([11], Proposition 5.3). Supposel coincides with $p$ and $\zeta_{p} \notin K$ or $l$ is an odd prime whose mod $p$ class generates $(\mathbf{Z} / p \mathbf{Z})^{*}$. Take any element $x$ in $\mathfrak{F}\left(D_{4 p}\right)$ of the form $x=\sum_{\chi \in \operatorname{Irr}^{-}} G, \chi(1)=2 x_{\chi} \mathrm{pr}_{\chi}$. Then for each fractional ideal $\mathfrak{A}$ of $K$ whose class in $C l(K)$ has l-power order,

- $\mathfrak{A}^{x w_{K} \theta_{K / K^{H}}}=(\alpha)$ for some $\alpha$ in $K^{\circ}$,

- $K\left(\alpha^{1 / w_{K, l}}\right)=K$,

where we set $H=\langle x\rangle$.

REMARK 5.3. The original statement of [11, Proposition 5.3] concerning the field $K\left(\alpha^{1 / w_{K, l}}\right)$ is that $K\left(\alpha^{1 / w_{K, l}}\right) / K^{H}$ is abelian. However, the author actually proved in [11] that $K\left(\alpha^{1 / w_{K, l}}\right)=K$ for primes $l$ as in Proposition 5.2.

Using this proposition, we prove the following result, which is our main result in this section.

THEOREM 5.4. Let $K / k$ be a finite Galois CM-extension whose Galois group is isomorphic to $D_{4 p}$ and let $S$ be a finite set of places of $k$ which contains $S_{\mathrm{ram}}$ and $S_{\infty}$. Then $B S(K / k, S, p)$ is true if $\zeta_{p} \notin K$ and $B S(K / k, S, l)$ is true for an odd prime $l$ whose mod $p$ class generates $(\mathbf{Z} / p \mathbf{Z})^{*}$.

Proof. Since $H$ is an abelian normal subgroup of $G$ with $[G: H]=2$, Conjecture 3.1 is true by Theorem 3.3. Therefore, by Theorem 4.4, we have only to show that $B S\left(K^{\mathrm{ab}} / k, S, l\right)$ is true and

$$
\mathfrak{A}^{d_{G} w_{K} \theta_{K / k, S}^{>1}}=(\alpha) \text { for some } \alpha \in K^{\circ} \text { and } K\left(\alpha^{1 / w_{k, l}}\right) / k \text { is strong central. }
$$

Since $\operatorname{Gal}\left(K^{\mathrm{ab}} / k\right)$ is $\mathbf{Z} / 2 \mathbf{Z} \times \mathbf{Z} / 2 \mathbf{Z}, B S\left(K^{\mathrm{ab}} / k, S, l\right)$ is true by [12, Theorem 2.1]. By Lemma 5.1, we have

$$
d_{G} w_{K} \theta_{K / k, S}^{>1}=\left(\sum_{\chi \in \operatorname{Irr}^{-} G, \chi(1)=2} \operatorname{pr}_{\chi}\right) \theta_{K / K^{H}, S_{H}} .
$$

Since the element $\sum_{\chi \in \operatorname{Irr}^{-}} G, \chi(1)=2 \mathrm{pr}_{\chi}$ is a central conductor as in Proposition 5.2, we obtain the statement (3) by Proposition 5.2. This completes the proof.

5.2. Extensions with group $Q_{2^{n+2}}$. Let $n$ be a nonzero natural number. In this subsection, we prove our main result for a CM-extension whose Galois group $G$ is isomorphic to $Q_{2^{n+2}}$. We use the presentation $Q_{2^{n+2}}=\left\langle x, y \mid x^{2^{n}}=y^{2}, x^{2^{n+1}}=1, y x y^{-1}=x^{-1}\right\rangle$. Then the center of $Q_{2^{n+2}}$ is $\left\{1, x^{2^{n}}\right\}$. 
5.2.1. Calculation of $d_{G}$ and Stickelberger elements. Conjugacy classes of $Q_{2^{n+2}}$ are $\{1\},\left\{x^{2^{n}}\right\},\left\{x^{2 k} y\right\}_{1 \leq k \leq 2^{n}},\left\{x^{2 k-1} y\right\}_{1 \leq k \leq 2^{n}}$ and $\left\{x^{l}, x^{-l}\right\}$, where $1 \leq l \leq 2^{n}-1$. Then we have

$$
\#\{1\}=\#\left\{x^{2^{n}}\right\}=1, \#\left\{x^{2 k} y\right\}_{1 \leq k \leq 2^{n}}=\#\left\{x^{2 k-1} y\right\}_{1 \leq k \leq 2^{n}}=2^{n} \text { and } \#\left\{x^{l}, x^{-l}\right\}=2 .
$$

Moreover, the commutator subgroup of $Q_{2^{n+2}}$ is the cyclic group $\left\langle x^{2}\right\rangle$ of order $2^{n}$. Combining these facts, we have

$$
d_{G}=1 . \mathrm{c} \cdot \mathrm{m}\left\{1,2,2^{n}\right\}=2^{n} .
$$

LEMMA 5.5. Let $K / k$ be a finite Galois CM-extension of number fields whose Galois group is isomorphic to $Q_{2^{n+2}}$ and let $S$ be a finite set of places of $k$ which contains $S_{\text {ram }}$ and $S_{\infty}$. We set $H:=\left\langle x^{2}\right\rangle$. Then

$$
d_{G} w_{K} \theta_{K / k, S}^{>1}=\left(\sum_{\chi \in \operatorname{Irr}^{-}} \operatorname{pr}_{\chi} \operatorname{pr}_{\chi(1)=2}\right) \frac{1}{2} \theta_{K / K^{H}, S_{H}} .
$$

Proof. Regarding $d_{G}$ as an element in $\mathbf{C}[G]$, we see that

$$
\begin{aligned}
d_{G}=2^{n} & =\sum_{\chi \in \operatorname{Irr} G} 2^{n} e_{\chi} \\
& =\sum_{\chi \in \operatorname{Irr} G, \chi(1)=1} 2^{n}\left(\frac{1}{2^{n+2}} \operatorname{pr}_{\chi}\right)+\sum_{\chi \in \operatorname{Irr} G, \chi(1)=2} 2^{n}\left(\frac{1}{2^{n+1}} \operatorname{pr}_{\chi}\right) \\
& =\sum_{\chi \in \operatorname{Irr}} \frac{1}{2^{2}, \chi(1)=1} \operatorname{pr}_{\chi}+\sum_{\chi \in \operatorname{Irr} G, \chi(1)=2} \frac{1}{2} \operatorname{pr}_{\chi} .
\end{aligned}
$$

Then by (2), we have

$$
\begin{aligned}
d_{G} w_{K} \theta_{K / k, S}^{>1} & =\left(\sum_{\chi \in \operatorname{Irr} G, \chi(1)=1} \frac{1}{2^{2}} \mathrm{pr}_{\chi}+\sum_{\chi \in \operatorname{Irr} G, \chi(1)=2} \frac{1}{2} \mathrm{pr}_{\chi}\right) w_{K}\left(1-\frac{1}{s_{G}} N_{[G, G]}\right) \theta_{K / K^{H}, S_{H}} \\
& =\left(\sum_{\chi \in \operatorname{Irr} G, \chi(1)=2} \frac{1}{2} \mathrm{pr}_{\chi}\right) w_{K} \theta_{K / K^{H}, S_{H}} \\
& =\left(\sum_{\chi \in \operatorname{Irr}^{-} G, \chi(1)=2} \frac{1}{2} \mathrm{pr}_{\chi}\right) w_{K} \theta_{K / K^{H}, S_{H}} \\
& =\left(\sum_{\chi \in \operatorname{Irr}^{-} G, \chi(1)=2} \operatorname{pr}_{\chi}\right) w_{K} \frac{1}{2} \theta_{K / K^{H}, S_{H}} .
\end{aligned}
$$

5.2.2. Main result. In this section we prove Theorem 5.8. We first prove the following, which is a slight refinement of [11, Proposition 5.8]. 
Proposition 5.6. Take any element $x$ in $\mathfrak{F}\left(Q_{2^{n+2}}\right)$ of the form $x=$ $\sum_{\chi \in \operatorname{Irr}^{-}} G, \chi(1)=2 x_{\chi} \mathrm{pr}_{\chi}$. Then for each fractional ideal $\mathfrak{A}$ of $K$ whose class in $C l(K)$ has 2-power order,

- $\mathfrak{A}^{x w_{K} \frac{1}{2} \theta_{K / K} H}=(\alpha)$ for some $\alpha$ in $K^{\circ}$,

- $K\left(\alpha^{1 / w_{K, 2}}\right)=K$,

where we set $H=\left\langle x^{2}\right\rangle$.

REMARK 5.7. The original statement of [11, Proposition 5.8] is

- $\mathfrak{A}^{x w_{K} \theta_{K / K} H}=(\alpha)$ for some $\alpha$ in $K^{\circ}$,

- $K\left(\alpha^{1 / w_{K, 2}}\right) / K^{H}$ is abelian.

Note that the factor $\frac{1}{2}$ before $\theta_{K / K^{H}}$ does not exist in the original claim.

Proof. We set $I_{K}^{+}:=\left\{\mathfrak{A} \mid \mathfrak{A}\right.$ is an ideal of $K$ and $\left.\mathfrak{A}^{j}=\mathfrak{A}\right\}$ and $A_{K}:=\operatorname{Coker}\left(I_{K}^{+} \rightarrow\right.$ $C l(K)) \otimes \mathbf{Z}_{2}$. We write $\phi$ for a generator of the group of characters of $\langle x\rangle$. Then a key point of the proof of [11, Proposition 5.8] is to prove the following inequality, which is (5.8) in [11]:

$$
\begin{aligned}
\left|A_{K}\right| & \leq \frac{4}{2^{2^{n}}} N_{\mathbf{Q}\left(\zeta_{2^{n+1}}\right) / \mathbf{Q}}\left(\frac{L\left(K / K^{H}, \phi, 0\right)}{2}\right) \\
& \leq N_{\mathbf{Q}\left(\zeta_{2^{n+1}}\right) / \mathbf{Q}}\left(\frac{L\left(K / K^{H}, \phi, 0\right)}{2}\right) .
\end{aligned}
$$

However, in fact, the first line of the above inequalities implies the following stronger inequality:

$$
\left|A_{K}\right| \leq N_{\mathbf{Q}\left(\zeta_{2^{n+1}}\right) / \mathbf{Q}}\left(\frac{L\left(K / K^{H}, \phi, 0\right)}{4}\right) .
$$

Then the same argument of the proof of [11, Proposition 5.8] implies that for each fractional ideal $\mathfrak{A}$,

$$
\mathfrak{A}^{\frac{1}{2} x \theta_{K / K^{H}}}=\left(\alpha^{\prime}\right)
$$

for some anti-unit $\alpha^{\prime}$. Hence we have

$$
\mathfrak{A}^{\frac{1}{2} x w_{K, 2} \theta_{K / K^{H}}}=\left(\alpha^{\prime w_{K, 2}}\right) .
$$

We set $\alpha:=\alpha^{\prime w_{K, 2}}$. Then we get the result as in Proposition 5.6.

Using this proposition, we prove the following, which is our main result in this section.

THEOREM 5.8. Let $K / k$ be a finite Galois CM-extension whose Galois group is isomorphic to $Q_{2^{n+2}}$ and let $S$ be a finite set of places of $k$ which contains $S_{\text {ram }}$ and $S_{\infty}$. Then $B S_{\mathrm{Gal}}(K / k, S, 2)$ is true. 
Proof. Since $H^{\prime}=\langle x\rangle$ is an abelian normal subgroup of $G$ with $\left[G: H^{\prime}\right]=2$, Conjecture 3.1 is true by Theorem 3.3. Therefore, by Theorem 4.4, we have only to show that $B S\left(K^{\mathrm{ab}} / k, S, 2\right)$ is true and

$$
\mathfrak{A}^{d_{G} w_{K} \theta_{K / k, S}^{>1}}=(\alpha) \text { for some } \alpha \in K^{\circ} \text { and } K\left(\alpha^{1 / w_{K, 2}}\right) / k \text { is strong central. }
$$

Since $\operatorname{Gal}\left(K^{\mathrm{ab}} / k\right)$ is $\mathbf{Z} / 2 \mathbf{Z} \times \mathbf{Z} / 2 \mathbf{Z}, B S\left(K^{\mathrm{ab}} / k, S, 2\right)$ is true by [12, Theorem 2.1]. By Lemma 5.5 , we have

$$
d_{G} w_{K} \theta_{K / k, S}^{>1}=\left(\sum_{\chi \in \operatorname{Irr}^{-}}^{G, \chi(1)=2} \mathrm{pr}_{\chi}\right) \frac{1}{2} \theta_{K / K^{H}}, S_{H} .
$$

Since the element $\sum_{\chi \in \operatorname{Irr}^{-}}$G, $\chi(1)=2 \mathrm{pr}_{\chi}$ is a central conductor as in Proposition 5.6, we obtain the statement (4) by Proposition 5.6. This completes the proof.

ACKNOWLEDGMENT. The author is grateful to the referee who gave valuable suggestions and comments and also pointed out the mistakes in the proof of Theorems 5.4 and 5.8 in the previous version of this paper. He also thanks to Masato Kurihara for his careful reading of earlier versions.

\section{References}

[ 1 ] D. Burns, On derivatives of Artin $L$-series, Invent. Math. 186 (2011), no. 2, 291-371.

[2] D. Burns, On derivatives of $p$-adic $L$-series at $s=0$, preprint, available at http://www.mth.kcl.ac.uk/staff/dj_burns/newdbpublist.html.

[ 3 ] C. W. CURTIS and I. REINER, Methods of Representation Theory with Applications to Finite Groups and Orders, Vol. 1, John Wiley \& Sons, 1981.

[4] G. Dejou and X.-F. Roblot, A Brumer-Stark conjecture for non-abelian Galois extensions, J. Number Theor. 142 (2014), 51-81.

[ 5 ] C. Greither, X.-F. Roblot and B. Tangedal, The Brumer-Stark Conjecture in some families of extensions of specified degree, Math. Comp. 73 (2004), no. 245, 297-315.

[ 6 ] C. Greither and C. PopesCU, An equivariant main conjecture in Iwasawa theory and applications, preprint, available at arXiv:1103.3069.

[ 7 ] H. JACOBINSKI, On extensions of lattices, Michigan Math. J. 13 (1966), 471-475.

[ 8 ] H. Johnston and A. NiCKel, Noncommutative fitting invariants and improved annihilation results, J. Lond. Math. Soc. 88 (2013), no. 1, 137-160.

[9] A. NiCKEL, On the equivariant Tamagawa number conjecture in tame CM-extensions, Math. Z. (268) (2010), no. $1-2,1-35$.

[10] A. NicKEL, On non-abelian Stark-type conjectures, Ann. Inst. Fourier 61 (2011), no. 6, 2577-2608.

[11] J. NOMURA, On non-abelian Brumer and Brumer-Stark conjecture for monomial CM-extensions, Int. J. Number Theory 10 (2014), no. 4, 817-848.

[12] J. SANDS, Galois groups of exponent two and the Brumer-Stark conjecture, J. Reine Angew. Math. 349 (1984), 129-135.

[13] J. TATE, Les Conjectures de Stark sur les Fonctions L d'Artin en s = 0, Birkhauser, 1984. 
Present Address

DePARTMENT OF MATHEMATICS,

KeIo University,

3-14-1 Hiyoshi, KонокU-KU, Yоконama, KanAGAWA 223-8522, JAPAN.

e-mail: nomura@math.keio.ac.jp 